\title{
SOME THEOREMS ON LACUNARY FOURIER SERIES, WITH EXTENSIONS TO COMPACT GROUPS
}

\author{
BY \\ EDWIN HEWITT AND H. S. ZUCKERMAN(')
}

1. Introduction. 1.1. The theory of lacunary Fourier series, developed by Banach, Kolmogorov, Sidon, Zygmund, and others, deals largely with extraordinary properties that such series possess. The Fourier series for a bounded function converges absolutely if the function has a lacunary Fourier series; an integrable function is square integrable if its Fourier series is lacunary. Somewhat different theorems show that very general functions on lacunary sets can be represented by Fourier or Fourier-Stieltjes transforms. Many of these theorems are given in [7, Chapter VI and Chapter IX].

In this paper, we use a method of Banach to show that various properties associated with lacunary series are in fact equivalent. Some degree of unity for different appearing facts is thus obtained. Fourier series are of such classical interest that we first treat them separately $(\$ \S 2-7)$. From our first equivalence theorem (2.1), we obtain new proofs of several theorems of Sidon (4.1), as well as new classes having some of the properties of lacunary series (Theorem 3.2 and \$5). Our second equivalence theorem (6.1) leads also to some new series possessing some of the properties of lacunary series (Theorems 7.1 and 7.3 and Example 7.4). In $\$ \$ 8$ and 9, we extend our results for Fourier series to an arbitrary compact group; these results of course contain those for Fourier series as special cases. Our interest in the problems discussed here arose from a remark of Professor Arne Beurling, which is a special case of Theorem 3.2. Since writing this paper, we have learned that several of our results have also been obtained independently by $\mathrm{R}$. E. Edwards. We wish to express our gratitude to Dr. Edwards for the privilege of sceing his manuscript.

1.2. Throughout the paper, "function" means "complex-valued function." In $\$ \S 2-7$, we are concerned with functions and measures on the interval $[0,2 \pi]$. Throughout these sections, the following notation is used. The symbols $\mathfrak{R}_{1}, \mathfrak{R}_{2}, \mathfrak{R}_{\infty}$ have their usual meanings, and norms $\|\cdot\|_{1},\|\cdot\|_{2},\|\cdot\|_{\infty}$, for Lebesgue measure and the interval $[0,2 \pi]$. The symbol $(5$ denotes the space of all continuous functions $x$ on $[0,2 \pi]$ such that $x(0)=x(2 \pi)$. It is normed with the uniform norm. The symbol $\mathfrak{T}$ denotes the space of all complex bounded Radon measures on $[0,2 \pi$ [. It is normed by the variation norm. For an integer $n, x \in \mathfrak{R}_{1}$, and $\mu \in \mathfrak{M}$, we define the Fourier transform $\hat{x}(n)$ by

Presented to the Society, January 21, 1959; received by the editors July 25, 1958.

(1) This paper was written with financial support from the National Science Foundation. 


$$
\hat{x}(n)=\frac{1}{2 \pi} \int_{0}^{2 \pi} e^{-i n t} x(t) d t
$$

and the Fourier-Stieltjes transform $\hat{\mu}(n)$ by

$$
\hat{\mu}(n)=\int_{[0,2 \pi[} e^{-i n t} d \mu(t) .
$$

The symbol $N$ is reserved for infinite sets of integers; our main Theorems 2.1 and 6.1 are trivial for finite sets. The symbol $c_{0}(N)$ denotes the space of all functions $\psi$ on $N$ such that $|\psi(n)| \geqq \alpha$ for only a finite subset of $N$, for all $\alpha>0$. This space is normed with the uniform norm. The symbol $l_{1}(N)$ denotes the space of all functions $\varphi$ on $N$ such that $\|\varphi\|_{1}=\sum_{n \in N}|\varphi(n)|<\infty$. The space $l_{2}(N)$ is defined similarly. The symbol $b(N)$ denotes the space of all bounded functions $f$ on $N$, with the norm $\|f\|=\sup _{n \in N}|f(n)|$. Given a function $f$ defined on $N$, we say that $f$ is matched by a function of a certain kind defined for all integers, say $g$, if $f(n)=g(n)$ for all $n \in N$.

2. First equivalence theorem. In this section, we show that four properties for sets of integers are equivalent.

2.1. Theorem. Let $N$ be a set of integers. The following properties of $N$ are equivalent.

(i) There is a positive number $A$ such that for every finite sequence $\left\{a_{1}, \cdots, a_{s}\right\}$ of complex numbers and every finite subset $\left\{n_{1}, \cdots, n_{s}\right\}$ of $N$, the inequality

$$
\sum_{k=1}^{s}\left|a_{k}\right| \leqq A \max _{0 \leqq t<2 \pi}\left|\sum_{k=1}^{s} a_{k} e^{i n_{k} t}\right|
$$

obtains.

(ii) If $x \in \mathbb{R}_{\infty}$ and $\hat{x}(m)=0$ for $m \in N$, then $\sum_{n \in N}|\hat{x}(n)|<\infty$.

(iii) For every function $\varphi \in c_{0}(N)$, there is a function $x \in \Omega_{1}$ such that $\hat{x}(-n)$ $=\varphi(n)$ for every $n \in N$.

(iv) For every function $f \in b(N)$, there is a measure $\mu \in \mathfrak{N}$ such that $\hat{\mu}(-n)$ $=f(n)$ for all $n \in N$.

REMARK. The equivalence of properties (i)-(iii), with only trivial changes, is due to Banach [2]. Property (iv) seems not to have been previously considered in this context. For the reader's convenience, we repeat the proof that (i), (ii), and (iii) are equivalent.

Proof of Theorem 2.1. Consider the Banach spaces $\mathfrak{l}_{1}$ and $c_{0}(N)$. The Fourier transform provides a linear mapping of $\mathfrak{l}_{1}$ into $c_{0}(N)$; this fact and its ramifications are the basis of the present proof. Specifically, we will use the mapping $\Phi$, defined for each $x \in \Omega_{1}$ as the function on $N$ such that

$$
\Phi(x)(n)=\hat{x}(-n)=\frac{1}{2 \pi} \int_{0}^{2 \pi} e^{i n t} x(t) d t \quad(n \in N) .
$$


The Riemann-Lebesgue lemma shows that the range of $\Phi$ is contained in $c_{0}(N)$, and it is also obvious that $\Phi$ is a bounded linear transformation of norm 1. The adjoint transformation $\Phi^{*}$ of $\Phi$ carries the adjoint space $l_{1}(N)$ of $c_{0}(N)$ into the adjoint space $\mathfrak{R}_{\infty}$ of $\mathfrak{R}_{1}$. If $f$ denotes the linear functional on $c_{0}(N)$ defined by an element $\psi$ of $l_{1}(N)$, then $\Phi^{*}(f)$ is by definition the linear functional on $\mathfrak{R}_{1}$ such that $\Phi^{*}(f)(x)=f(\Phi(x))$ for all $x \in \mathfrak{R}_{1}$. Thus for $\psi \in l_{1}(N)$, we have

$$
\begin{aligned}
\frac{1}{2 \pi} \int_{0}^{2 \pi} x(t) \Phi^{*}(\psi)(t) d t & =\sum_{n \in N} \Phi(x)(n) \psi(n)=\sum_{n \in N}\left(\frac{1}{2 \pi} \int_{0}^{2 \pi} x(t) e^{i n t} d t\right) \psi(n) \\
& =\frac{1}{2 \pi} \int_{0}^{2 \pi} x(t)\left(\sum_{n \in N} \psi(n) e^{i n t}\right) d t .
\end{aligned}
$$

Therefore

$$
\Phi^{*}(\psi)(t)=\sum_{n \in N} \psi(n) e^{i n t} .
$$

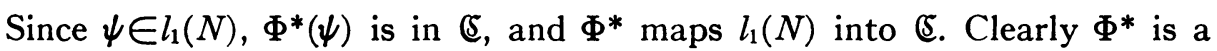
bounded, one-to-one, linear transformation of norm 1 .

Consider now the following property:

(v) $\Phi^{*-1}$ is bounded.

Property (v) may be restated as: there is a positive number $A$ such that

$$
\sum_{n \in N}|\psi(n)| \leqq A \max _{0 \leqq i<2 \pi}\left|\sum_{n \in N} \psi(n) e^{i n t}\right|
$$

for all $\psi \in l_{1}(N)$. Plainly this is equivalent to (i), so that (i) and (v) are equivalent.

We next show that (i) and (ii) are equivalent. Suppose that (i) holds. For $x \in R_{\infty}$, we have

$$
\max _{0 \leqq t<2 \pi}\left|\sum_{k=-\infty}^{p}\left(1-\frac{|k|}{p+1}\right) \hat{x}(k) e^{i k t}\right| \leqq\|x\|_{\infty}
$$

for all positive integers $p$. If $\hat{x}(m)=0$ for $m \notin N$ and (i) holds, it follows that

$$
\sum\left(1-\frac{|k|}{p+1}\right)|\hat{x}(k)| \leqq A \cdot\|x\|_{\infty},
$$

for all positive integers $p$, the sum being taken over the set

$$
N \cap\{-p,-p+1, \cdots, p-1, p\} .
$$

Property (ii) follows at once. Suppose now that (ii) holds. We shall show that $\Phi^{*}\left(l_{1}(N)\right)$ is a closed subspace of $\mathbb{E}$, and from this a theorem of Banach $\left.{ }^{2}\right)$

(2) This and the two following theorems quoted from Banach are stated by him for real Banach spaces. The proofs for the complex case needed here are unchanged. 
$[1$, p. 41 , Théorème 4$]$, will show that $\Phi^{*-1}$ is continuous. Let $\left\{\psi_{n}\right\}_{n=1}^{\infty}$ be a sequence of elements of $l_{1}(N)$ such that $\lim _{n \rightarrow \infty} \Phi^{*}\left(\psi_{n}\right)=y$ exists in $\mathcal{E}^{*}$. It is easy to see that $\hat{y}(m)=0$ for $m \notin N$, as this is true of all $\Phi^{*}\left(\psi_{n}\right)$. Hence $\sum_{n=-\infty}^{\infty}|\hat{y}(n)|$ is finite, and $y(t)=\sum_{n \in N} \hat{y}(n) e^{i n t}$. That is, $y \in \Phi^{*}\left(l_{1}(N)\right)$, and $\Phi^{*}\left(l_{1}(N)\right)$ is a closed subspace of $\mathfrak{C}$.

The equivalence of (i) and (iii) is proved by appealing to another theorem of Banach [1, p. 146, Théorème 1 and p. 148, Théorème 4]: $\Phi$ maps $\Omega_{1}$ onto $c_{0}(N)$ if and only if $\Phi^{*-1}$ is continuous.

Finally, to prove the equivalence of (i) and (iv), we construct the linear transformation $\Phi^{* *}$, whose domain is the adjoint space $\mathscr{T}$ of $\mathfrak{E}$, and whose range is contained in the adjoint space $b(N)$ of $l_{1}(N)$. For $\mu \in \mathbb{T}$, we find as in computing $\Phi^{*}$ that $\Phi^{* *}(\mu)(n)=\int_{[0,2 \pi[} e^{i n t} d \mu(t)$ for all $n \in N$. A third theorem of Banach [1, p. 147, Théorème 2 and p. 148, Théorème 3$]$ shows that $\Phi^{* *}$ maps $\mathfrak{T}$ onto $b(N)$ if and only if $\Phi^{*-1}$ is continuous. This completes the proof.

2.2. Remark. It is obvious that 2.1 (i) is equivalent to the following condition.

(i') There is a positive number $A$ such that for every finite sequence $\left\{a_{1}, \cdots, a_{s}\right\}$ of complex numbers and every finite subset $\left\{n_{1}, \cdots, n_{s}\right\}$ of $N$, the inequality

$$
\sum_{k=1}^{s}\left|a_{k}\right| \leqq A \max _{0 \leqq t<2 \pi}\left|\sum_{k=1}^{s} a_{k} e^{-i n k t}\right|
$$

obtains.

Let $\Phi^{\prime}$ be the mapping of $\mathfrak{l}_{1}$ into $c_{0}(N)$ such that $\Phi^{\prime}(x)(n)=\hat{x}(n)$. Then we can repeat the proof of Theorem 2.1, mutatis mutandis, and show that (i)-(iv) are equivalent to $\left(\mathrm{i}^{\prime}\right)-\left(\mathrm{iv}^{\prime}\right)$, where $\left(\mathrm{ii}^{\prime}\right)$ is obtained from (ii) by replacing $\hat{x}(m)$ by $\hat{x}(-m)$, (iii') from (iii) by replacing $\hat{x}(-n)$ by $\hat{x}(n)$, and (iv') from (iv) by replacing $\hat{\mu}(-n)$ by $\hat{\mu}(n)$. For example, (iii) and (iii') are equivalent, so that every function in $c_{0}(N)$ is matchable by a Fourier transform $\hat{x}(n)$ if and only if every such function is matchable by a Fourier transform $\hat{x}(-n)$. Obviously too all eight assertions (i)-(iv') are equivalent.

3. A sufficient condition for Theorem 2.1. In this section, we show that every set of integers enjoying a certain independence condition satisfies all of the conditions of Theorem 2.1. Curiously enough, condition 2.1 (iv) is an easy one to deal with. We require a lemma.

3.1. Lemma. Let $N$ be a set of integers, enumerated in any way: $N$ $=\left\{n_{1}, n_{2}, n_{3}, \cdots\right\}$. Let $f$ be a bounded function on $N$. Suppose that for an infinite set $S$ of positive integers $s$, there is a function $h_{s} \in \Omega_{1}$ such that $\hat{h}_{s}\left(-n_{k}\right)$ $=f\left(n_{k}\right) \quad(k=1,2, \cdots, s)$ and such that $(1 / 2 \pi) \int_{0}^{2 \pi}\left|h_{s}(t)\right| d t$ is bounded on $S$. Then there is a measure $\mu \in \mathbb{N}$ such that $\hat{\mu}\left(-n_{k}\right)=f\left(n_{k}\right)$ for $k=1,2,3, \cdots$. The measure $\mu$ can be taken non-negative if all of the functions $h_{s}$ are non-negative.

Proof. The functions $h_{s}$ can be identified with elements $\nu_{s}$ of $\mathscr{T}$, in the usual way: $\nu_{s}(A)=(1 / 2 \pi) \int_{\Lambda} h_{s}(t) d t$ for Borel sets $A$. The space $\mathfrak{T}$ is a concrete 
representation of the adjoint space $\mathfrak{E}^{*}$ of $\mathfrak{E}$, and the norm $\left\|\nu_{s}\right\|$ of $\nu_{s}$ as an element of $\mathbb{E}^{*}$ is $(1 / 2 \pi) \int_{0}^{2 \pi}\left|h_{s}(t)\right| d t$. Our hypotheses imply that $\left\{\left\|\nu_{s}\right\|\right\}_{s=1}^{\infty}$ is bounded. Hence the set $\left\{\nu_{s}\right\}_{s=1}^{\infty}$ has at least one weak limit point in $\mathfrak{T}$, say $\mu$. (If all $h_{s}$ are non-negative, the measure $\mu$ is necessarily non-negative.) For this $\mu$, any positive integer $k$, and any positive number $\epsilon$, there are an infinite number of positive integers $s$ such that

$$
\left|\int_{[0,2 \pi l} e^{i n k t} d \mu(t)-\frac{1}{2 \pi} \int_{0}^{2 \pi} e^{i n k t} h_{s}(t) d t\right|<\epsilon .
$$

This implies that $\int_{[0,2 \pi[} e^{i n_{k} t} d \mu(t)=f\left(n_{k}\right)$ for $k=1,2,3, \cdots$, and this proves the lemma.

3.2. THEOREM. Let $N$ be a set of integers, and suppose that $N$ can be written as the union of a finite number of pairwise disjoint infinite sets $N_{\mathrm{r}}, \cdots, N_{r}$ satisfying the following condition. For every nonvoid finite subset $\left\{n_{1}, \cdots, n_{\mathrm{s}}\right\}$ of every $N_{j}$, and every sum $\sigma=\sum_{k=1}^{s} \epsilon_{k} n_{k}$, where each $\epsilon_{k}$ can be \pm 1, no $\sigma$ is zero, and $\sigma$ belongs to $N$ only if $s=1, \epsilon_{1}=1$, and $\sigma=n_{1}$. Then $N$ satisfies the conditions of Theorem 2.1.

Proof. Consider a bounded function $f$ on $N$. We wish to show that there is a measure $\mu \in \mathscr{N}$ such that $\hat{\mu}(-n)=f(n)$ for all $n \in N$. Clearly we may suppose that $|f(n)| \leqq 1$ for all $n \in N$. Order each set $N_{j}$ arbitrarily: $N_{j}$ $=\left\{n_{1}^{(j)}, n_{2}^{(j)}, n_{3}^{(j)}, \cdots\right\}$. We first note that $-n_{k}^{(j)} \notin N$ for all $k$ and $j$ and that $0 \notin N$. For every positive integer $u$ and every $j, 1 \leqq j \leqq r$, let

$$
h_{u}^{(j)}(t)=2 \prod_{k=1}^{u}\left(1+\Re\left(f\left(n_{k}^{(j)}\right) e^{-i n_{k}^{(j) t}}\right)\right) .
$$

Clearly each $h_{u}^{(j)}$ is a non-negative function. Multiplying out, we have $\left(^{(3)}\right.$

$$
h_{u}^{(j)}(t)=2+\sum_{k=1}^{u}\left(f\left(n_{k}^{(j)}\right) e^{-i n_{k}^{(j) t}}+\bar{f}\left(n_{k}^{(j)}\right) e^{i n_{k}^{(j) t}}\right)+\sum_{\cdot} a_{\sigma} e^{i \sigma t},
$$

where every $\sigma$ appearing in the second sum has the form $\sigma=\sum \epsilon_{l} n_{l}^{(j)}$, summed over two or more $l$ 's, $1 \leqq l \leqq u$, and where the $\epsilon_{l}$ 's are \pm 1 . Multiplying 3.2.1 by $(1 / 2 \pi) e^{i n t}$ and integrating from 0 to $2 \pi$, we obtain

$$
\hat{h}_{u}^{(j)}(-n)=\left\{\begin{array}{cl}
f\left(n_{k}^{(j)}\right) & \text { if } n=n_{k}^{(j)}, \quad 1 \leqq k \leqq u, \\
0 & \text { if } n \in N \cap N_{j}^{\prime}, \\
2 & \text { if } n=0 .
\end{array}\right.
$$

For $u=1,2,3, \cdots$, let $h_{r u}$ be the function $h_{r u}=\sum_{y-1}^{r} h_{u}^{(f)}$. Plainly $\left|h_{r u}\right|=\sum_{j=1}^{r} h_{u}^{(j)}$, and $f(x)$.

(ग) For typographical reasons, we write $\bar{f}(x)$ to mean the complex conjugate of the number 


$$
\frac{1}{2 \pi} \int_{0}^{2 \pi}\left|h_{r u}(t)\right| d t=\sum_{j=1}^{k} \hat{h}_{u}^{(j)}(0)=2 r .
$$

Furthermore, we have

$$
\hat{h}_{r u}\left(-n_{k}\right)=\sum_{j=1}^{r} \hat{h}_{u}^{(j)}\left(-n_{k}^{(p)}\right)=f\left(n_{k}^{(p)}\right)
$$

if $1 \leqq k \leqq u$ and $1 \leqq p \leqq r$. Thus the functions $h_{r u}(u=1,2,3, \cdots)$ satisfy all of the conditions of 3.1 , with $S=\{r, 2 r, 3 r, \cdots\}$. Applying 3.1, we have 2.1 (iv), and hence the other conditions of Theorem 2.1 as well.

4. A proof of two theorems of Sidon. Let $N$ be a set of positive integers, $N=\left\{n_{1}, n_{2}, n_{3}, \cdots\right\}$, such that $n_{k+1} / n_{k} \geqq \lambda>1$ for $k=1,2,3, \cdots$. Sidon has proved (in slightly altered form) that $N$ satisfies conditions 2.1 (ii) and 2.1 (iv) (see [7, pp. 139 and 220]). We now prove Sidon's theorems from Theorems 2.1 and 3.2.

4.1. TheOREM. Let $N=\left\{n_{1}, n_{2}, n_{3}, \cdots\right\}$ be a set of positive integers such that $n_{k+1} / n_{k} \geqq \lambda>1$ for $k=1,2,3, \cdots$. Then $N$ satisfies all of the conditions of Theorem 2.1.

Proof. We will show that $N$ satisfies the conditions of Theorem 3.2. Choose a positive integer $r$ such that

$$
\lambda^{r}>\frac{2 \lambda-1}{\lambda-1}
$$

and let $N_{j}=\left\{n_{j}, n_{r+j}, n_{2 r+j}, \cdots\right\}, 1 \leqq j \leqq r$. For convenience, we write the sums $\sigma$ of Theorem 3.2 in the form $\sigma=\sum_{k=0}^{s} \delta_{k} n_{r k+j}$, where each $\delta_{k}$ can be 1,0 , or -1 , and $1 \leqq j \leqq r$. Now writing $\tau=\sum_{k=0}^{s-1} \delta_{k} n_{r k+j}$, we have

$$
|\tau| \leqq \sum_{k=0}^{s-1} n_{r k+j} \leqq \sum_{k=0}^{s-1} n_{r(s-1)+j} \lambda^{-r(s-1-k)}
$$

since $n_{l} \leqq \lambda^{-v} n_{l+v}$. Hence we have

$$
|\tau|<n_{r(8-1)+j} \sum_{l=0}^{\infty} \lambda^{-r l}=\frac{\lambda^{r}}{\lambda^{r}-1} n_{r(s-1)+j} \leqq \frac{1}{\lambda^{r}-1} n_{r s+j} .
$$

From 4.1.2 and 4.1.1, we have

$$
|\tau|<n_{r s+j}
$$

If any $\sigma$ as in Theorem 3.2 were 0 , we could write $0=\sum_{k=0}^{s} \delta_{k} n_{r k+j}$ with $\delta_{s}= \pm 1$. This would contradict 4.1.3. Thus the first requirement on the $\sigma$ 's in Theorem 3.2 is met. 
To verify the second requirement on the $\sigma$ 's in Theorem 3.2, suppose that $n_{p}=\sum_{k=0}^{s} \delta_{k} n_{r k+j}$, where we may suppose that $\delta_{\varepsilon}= \pm 1$. The inequality 4.1.3 shows that $\delta_{s}=1$, since $n_{p}$ is positive. From 4.1.2 we have

4.1 .4

$$
\left|n_{p}-n_{r s+j}\right|<\frac{1}{\lambda^{r}-1} n_{r s+j} .
$$

We wish to show that $p=r s+j$. If $p>r s+j$, then $n_{p} \geqq \lambda n_{r s+j}$, and hence $\left|n_{p}-n_{r s+j}\right|=n_{p}-n_{r s+j} \geqq(\lambda-1) n_{r s+j}$. This inequality and 4.1.4 imply that $(\lambda-1)<\left(\lambda^{r}-1\right)^{-1}$, which contradicts 4.1.1. If $p<r s+j$, then $n_{p} \leqq \lambda^{-1} n_{r s+j}$, and hence $\left|n_{p}-n_{r s+j}\right|=n_{r s+j}-n_{p} \geqq\left(1-\lambda^{-1}\right) n_{r s+j}$. This inequality and 4.1.4 imply that $\left(1-\lambda^{-1}\right)<\left(\lambda^{r}-1\right)^{-1}$, which contradicts 4.1 .1 . These contradictions imply that $p=r s+j$. Therefore we have $0=\sum_{k=0}^{s-1} \delta_{k} n_{r k+j}$, and $\delta_{0}=\cdots=\delta_{s-1}$ $=0$, since, as we have already proved, the $\sigma$ 's satisfy the first requirement of Theorem 3.2. Since $\delta_{s}=1$, the second requirement of Theorem 3.2 is satisfied. The proof is completed by applying Theorem 2.1 .

4.2. Remark. Sidon has proved [5] that 2.1 (ii) holds for a set of integers $N$ that can be written in the form $\bigcup_{j=1}^{r} L_{j}$, where $x, y \in L_{j}$ and $x>y$ imply that $x>\lambda_{j} y\left(\lambda_{j}>1\right)$. Thus all of the conditions of Theorem 2.1 hold for such sets.

5. Remarks and examples for Theorem 2.1.

5.1. THEOREM. Let $N$ be a set of integers satisfying the conditions of Theorem 2.1, and let $S$ and $T$ be finite sets of integers. Then $\left(N \cap S^{\prime}\right) \cup T$ also satisfies the conditions of Theorem 2.1.

Proof. Let $T \cap N^{\prime}=\left\{n_{1}, \cdots, n_{u}\right\}$, let $\alpha_{1}, \cdots, \alpha_{u}$ be complex numbers, and $x(t)=\sum_{j=1}^{u} \alpha_{j} e^{-i n_{j} t}$. Then $\hat{x}\left(-n_{j}\right)=\alpha_{j}(j=1, \cdots, u)$ and $\hat{x}(n)=0$ for all other values of $n$. Hence $N \cup T$ satisfies 2.1 (iii). Since $\left(N \cap S^{\prime}\right) \cup T \subset N \cup T$, the present theorem is proved.

5.2. TheOREM. Let $N$ be a set of integers satisfying the conditions of Theorem 2.1, let a be any integer, and $N+a$ the translate of $N$ by $a$. Then $N+a$ satisfies the conditions of Theorem 2.1.

Proof. Let $x \in \mathfrak{R}_{1}$ and $x_{a}(t)=x(t) e^{i a t}$. Then $\hat{x}_{a}(-n)=\hat{x}(-(n+a))$. Hence $N+a$ satisfies 2.1 (iii) if $N$ does.

5.3. Theorem. Let $N$ be a set of integers satisfying the conditions of Theorem 2.1 and let $-N=\{-n\}_{n \in N}$. Then $-N$ satisfies the conditions of Theorem 2.1.

Proof. This is implicit in 2.2 and is also obvious from 2.1 (i).

5.4. REMARK. Let $N$ be any set of integers satisfying the conditions of Theorem 3.2. All of the functions $h_{r u}$ constructed in the proof of Theorem 3.2 are non-negative, and hence, as noted in Lemma 3.1, every bounded function on $N$ can be matched by a function $\hat{\mu}(-n)$, where $\mu$ is a non-negative measure on $[0,2 \pi[$. (In other words, every bounded function $f$ on $N$ can be extended 
to a positive definite function $f_{1}$ defined on the set of all integers; it follows from the proof of Theorem 3.2 that $f_{1}(0) \leqq 2 r \sup _{n \in N}|f(n)|$.) For every real measure $\mu, \hat{\mu}(n)$ is the complex conjugate of $\hat{\mu}(-n)$, and hence a set $N$ with this property can contain no integer and its negative.

5.5. ThEOREM. Let $N$ be a set of integers such that every bounded function on $N$ can be matched by a function $\hat{\mu}(-n)$, where $\mu$ is a real measure. Then $N \cup(-N)$ satisfies the conditions of Theorem 2.1.

Proof. Let $f$ be any bounded function on $N \cup(-N)$ (note that $N \cap(-N)$ is void). Let

$$
\begin{aligned}
& f_{1}(n)=\frac{1}{2}(f(n)+\bar{f}(-n)), \\
& f_{2}(n)=\frac{1}{2 i}(f(n)-\bar{f}(-n)),
\end{aligned}
$$

for $n \in N$. Let $\mu_{j}$ be a real measure in $\mathfrak{M}$ such that $\boldsymbol{\mu}_{j}(-n)=f_{j}(n)(n \in N, j=1,2)$, and let $\mu=\mu_{1}+i \mu_{2}$. Then it is easy to see that $\hat{\mu}(-n)=f(n)$ for $n \in N \cup(-N)$.

5.6. EXAMPLES. It is of some interest to note that there are sets satisfying the conditions of Theorem 3.2 (and hence of Theorem 2.1) that do not have the lacunary property of Theorem 4.1. Let $a$ be any integer greater than 1 . The set $\left\{a^{k}, a^{k}+1\right\}_{k=1}^{\infty}$ satisfies the conditions of Theorem 3.2; it does not satisfy the lacunary property but is of course the union of two such sets. (See also 4.2.)

There are also sets $N$ satisfying the conditions of Theorem 3.2 that cannot be written as the union of any finite number of sets with the lacunary property of Theorem 4.1. Let $N$ be the set of all numbers $3^{2 l+2}+3^{k}$, where $k=2^{l}, 2^{l}+1$, $2^{l}+2, \cdots, 2^{l+1}-1$, and $l=0,1,2, \cdots$. For each positive integer $k$, there is a unique non-negative integer $l$ such that $2^{l} \leqq k<2^{l+1}$. Hence every exponent $k$ appears exactly once in a number belonging to $N$, and we may write $n_{k}=3^{2^{l+2}}+3^{k}(k=1,2,3, \cdots)$. We show that $N$ satisfies the conditions of Theorem 3.2, with $r=1$. For convenience, we write the sums $\sigma$ of Theorem 3.2 in the form $\sigma=\sum_{k=1}^{s} \delta_{k} n_{k}$, where each $\delta_{k}$ can be 1,0 , or -1 . Assume that $\sigma=0$ and that some $\delta_{k} \neq 0$. Let $k_{0}$ be the smallest $k$ such that $\delta_{k} \neq 0$. For $k>k_{0}$, we see that $n_{k} \equiv 0\left(\bmod 3^{k_{0}+1}\right)$ and that $n_{k_{0}} \equiv 3^{k_{0}}\left(\bmod 3^{k_{0}+1}\right)$. Therefore $\delta_{k_{0}} 3^{k_{0}} \equiv 0\left(\bmod 3^{k_{0}+1}\right)$ and hence $\delta_{k_{0}} \equiv 0(\bmod 3)$, which contradicts the definition of $\delta_{k_{0}}$. Suppose that $\sigma=n_{p}$ and that $k_{0}$ is the smallest $k$ such that $\delta_{k} \neq 0$. Then $\delta_{k_{0}} 3^{k_{0}} \equiv n_{p}\left(\bmod 3^{k_{0}+1}\right)$, which implies that $p \geqq k_{0}$, and hence $\delta_{k_{0}}$ $\equiv 3^{p-k_{0}}(\bmod 3)$. From this we see that $p=k_{0}$ and $\delta_{k_{0}}=1$. Consequently $\sigma^{\prime}=\sigma-\delta_{k_{0}} n_{k_{0}}=0$, and hence every $\delta_{k}$ appearing in $\sigma^{\prime}$ is 0 . Thus $N$ satisfies the conditions of Theorem 3.2.

Assume that $N$ can be written as a union $\bigcup_{j=1}^{r} L_{j}$, where each $L_{j}$ is a lacunary set: $x, y \in L_{j}$ and $x>y$ imply that $x \geqq \lambda_{j} y$, where $\lambda_{j}>1$. Let $\lambda$ 
$=\min \left(\lambda_{1}, \cdots, \lambda_{r}\right)$. Let $2^{k}$ be greater than $r$. Then at least two of the numbers $n_{2^{k}}, n_{2^{k+1}}, \cdots, n_{2^{k+1}-1}$ lie in a single $L_{j}$. Suppose that these are $x$ and $y$, $x>y$. Then $n_{2^{k+1-1}} \geqq x \geqq \lambda y \geqq \lambda n_{2^{k}}$, and

$$
\lambda \leqq \frac{n_{2^{k+1}-1}}{n_{2^{k}}}=\frac{3^{2 k+2}+3^{2 k+1-1}}{3^{2 k+2}+3^{2 k}}<1+3^{-2^{k+1}} .
$$

For $k$ sufficiently large, this contradicts the inequality $\lambda>1$. In this example, 3 can be replaced by any larger integer.

6. Second equivalence theorem. We prove in this section a theorem somewhat like Theorem 2.1. Once again, our methods are based on Banach [2].

6.1. THEOREM. Let $N$ be a set of integers. The following conditions on $N$ are equivalent.

(i) There is a positive number $B$ such that for every finite sequence $\left\{a_{1}, \cdots, a_{s}\right\}$ of complex numbers and every finite subset $\left\{n_{1}, \cdots, n_{s}\right\}$ of $N$, the inequality

$$
\left(\sum_{k=1}^{s}\left|a_{k}\right|^{2}\right)^{1 / 2} \leqq B \frac{1}{2 \pi} \int_{0}^{2 \pi}\left|\sum_{k=1}^{s} a_{k} e^{i n k t}\right| d t
$$

obtains.

(ii) If $x \in \mathfrak{R}_{1}$ and $\hat{x}(m)=0$ for $m \notin N$, then $\sum_{n=-\infty}^{\infty}|\hat{x}(n)|^{2}<\infty$.

(iii) For every function $\varphi \in l_{2}(N)$, there is a function $x \in \mathbb{C}$ such that $\hat{x}(-n)$ $=\varphi(n)$ for all $n \in N$.

(iv) For every function $\varphi \in l_{2}(N)$, there is a function $x \in \mathbb{R}_{\infty}$ such that $\hat{x}(-n)$ $=\varphi(n)$ for all $n \in N$.

Proof. The proof is very like that of Theorem 2.1. We consider the spaces $\sqrt{5}$ and $l_{2}(N)$, and the mapping $\Phi$ of $\mathbb{E}$ into $l_{2}(N)$ such that $\Phi(x)(n)=\hat{x}(-n)$ for all $n \in N$ and $x \in \mathbb{E}$. The mapping $\Phi$ is clearly linear and has norm 1 . The adjoint mapping $\Phi^{*}$ carries $l_{2}(N)$ into $\mathscr{T}$. For $\varphi \in l_{2}(N)$, it is easy to see that $\Phi^{*}(\varphi)$ is an absolutely continuous measure, whose derivative is the function $\sum_{n \in N} \varphi(n) e^{i n t}$, which is in $\mathfrak{R}_{2}$. Thus we have $\Phi^{*}\left(l_{2}(N)\right) \subset \mathfrak{R}_{2} \subset \mathfrak{R}_{1}$. We omit the details.

The implication (i) $\Rightarrow$ (ii) is proved as in Theorem 2.1 , with the observation that the Fejér sums

$$
\sum_{k=-p}^{p}\left(1-\frac{|k|}{p+1}\right) \hat{x}(k) e^{i k t}
$$

converge in the $\mathbb{R}_{1}$ norm to $x$, for every $x \in \mathfrak{R}_{1}$. The implication (ii) $\Rightarrow(\mathrm{i})$ and the equivalence of (i) and (iii) are proved just as in Theorem 2.1.

To prove that (iii) and (iv) are equivalent, we construct the mapping $\Phi^{* *}$, which carries the adjoint space $\mathfrak{R}_{\infty}$ of $\ell_{1}$ into the adjoint space $l_{2}(N)$ of $l_{2}(N)$. For $x \in \mathbb{R}_{\infty}$ and $n \in N$, we have $\Phi^{* *}(x)(n)=\hat{x}(-n)$. The equivalence of (i) and (iv) is now proved just as in Theorem 2.1. 
6.2. Remark. We can repeat Remark 2.2 with suitable changes and obtain four more assertions equivalent to 6.1 (i) -6.1 (iv). For example, the condition

(iii') for every $\varphi \in l_{2}(N)$, there is a function $x \in \mathbb{E}$ such that $\hat{x}(n)=\varphi(n)$ for all $n \in N$

is equivalent to 6.1 (i)-6.1 (iv).

6.3. Remark. The mapping $x \rightarrow \hat{x}(-n)$ is a bounded linear transformation carrying $\mathbb{E}$ into $l_{p^{\prime}}(N)$, where $1<p \leqq 2$ and $p^{\prime}=p /(p-1)$. Hence we might try to generalize Theorem 6.1 by using an arbitrary $l_{p^{\prime}}(N)$ instead of $l_{2}(N)$. Conditions 6.1 (i)-6.1 (iv) have obvious analogues, and they are all equivalent. However, the analogue of 6.1 (iii) states that for every $\varphi \in l_{p^{\prime}}(N)$, there is a function $x \in \mathbb{E}$ such that $\hat{x}(-n)=\varphi(n)$ for all $n \in N$. This implies that $l_{p^{\prime}}(N) \subset l_{2}(N)$, an inclusion which obtains only if $N$ is finite (a case devoid of interest) or $p^{\prime}=p=2$.

\section{Some sufficient conditions for Theorem 6.1.}

7.1. Theorem. Let $N$ be a set of integers. Suppose that for every integer $m$, the number of solutions of the equation $x+y=m$ with $x, y \in N$ has a finite upper bound $B$ independent of $m$. Then $N$ satisfies the conditions of Theorem 6.1.

Proof. Let $\left\{n_{1}, n_{2}, \cdots, n_{s}\right\}$ be any nonvoid finite subset of $N$, $\left\{a_{1}, \cdots, a_{s}\right\}$ any sequence of complex numbers not all zero, and $P(t)$ $=\sum_{k=1}^{s} a_{k} e^{i n_{k t}}$. Then we have $P(t)^{2}=\sum c_{m} e^{i m t}$, where $c_{m}=\sum a_{k} a_{l}$, summed over the set $T(m)$ of all pairs $(k, l)$ such that $n_{k}+n_{l}=m, 1 \leqq k \leqq s, 1 \leqq l \leqq s$. Thus $\left|c_{m}\right| \leqq \sum\left|a_{k}\right| \cdot\left|a_{l}\right| \leqq B \max \left\{\left|a_{k}\right| \cdot\left|a_{l}\right|:(k, l) \in T(m)\right\}$ and

$$
\left|c_{m}\right|^{2} \leqq B^{2} \sum_{(k, l) \in T(m)}\left|a_{k}\right|^{2}\left|a_{l}\right|^{2} .
$$

We then have

$$
\frac{1}{2 \pi} \int_{0}^{2 \pi}|P(t)|^{4} d t=\sum\left|c_{m}\right|^{2} \leqq B^{2} \sum_{m} \sum_{(k, l) \in T(m)}\left|a_{k}\right|^{2}\left|a_{l}\right|^{2}
$$

$$
\leqq B^{2} \sum_{k=1}^{s} \sum_{l=1}^{s}\left|a_{k}\right|^{2}\left|a_{l}\right|^{2}=B^{2}\left(\sum_{k=1}^{n}\left|a_{k}\right|^{2}\right)^{2}=B^{2}\left(\frac{1}{2 \pi} \int_{0}^{2 \pi}|P(t)|^{2} d t\right)^{2} \text {. }
$$

Hölder's inequality with $p=3$ gives the inequality

$$
\frac{\int x^{2}}{\left(\int x\right)^{2}} \leqq \frac{\int x^{4}}{\left(\int x^{2}\right)^{2}},
$$

if $x$ is non-negative. Taking $x=|P(t)|$, we infer from 7.1.1 that

$$
\left(\sum_{k=1}^{s}\left|a_{k}\right|^{2}\right)^{1 / 2} \leqq B \frac{1}{2 \pi} \int_{0}^{2 \pi}|P(t)| d t .
$$


This is 6.1 (i), and the proof is complete.

7.2. REmarks. Sidon has proved a somewhat weakened version of Theorem 7.1, in [6].

We note that Theorems 5.1, 5.2, 5.3 and 5.5 have evident analogues relating to Theorem 6.1. We next extend another theorem of Sidon and Banach [7, p. 215].

7.3. Theorem. Let $N$ be a set of positive integers such that $N=\bigcup_{j=1}^{r} L_{j}$, where each $L_{j}$ satisfies a lacunary condition: $x, y \in L_{j}$ and $x>y$ imply that $x \geqq \lambda_{j} y$, where $\lambda_{j}>1$. Then $N$ satisfies all of the conditions of Theorem 6.1.

Proof. We will show that $N$ satisfies the condition of Theorem 7.1. For an integer $m$ of the form $u+v$ with $u, v \in N$, we consider all pairs $(x, y)$ such that $(x, y) \in N, x \geqq y$, and $x+y=m$. Of all these pairs, let $(p, q)$ be the one with the smallest value of $p$. We then have $p \leqq x<x+y=p+q \leqq 2 p$. Thus we have $p \leqq x<2 p$. Let $x_{1}, \cdots, x_{k}$, with $x_{1}<x_{2}<\cdots<x_{k}$, be all such $x$ 's that are in a fixed $L_{j}$. Then we have $2 p>x_{k} \geqq \lambda_{j}^{k-1} x_{1} \geqq \lambda_{j}^{k-1} p$. It follows that $k<\log 2 / \log \lambda_{j}+1$. Thus the number of pairs $(x, y)$ is less than $r+\sum_{j=1}^{r} \log 2 / \log \lambda_{j}$, and the number of solutions in $N$ of the equation $u+v=m$ is less than $2 r+2 \sum_{j=1}^{r} \log 2 / \log \lambda_{j}$, which can thus be taken as the number $B$ of Theorem 7.1.

7.4. ExAmple. It is of interest to give an example of a set $N$ $=\left\{n_{1}, n_{2}, n_{3}, \cdots\right\}$ satisfying the condition of Theorem 7.1 that is not a finite union of lacunary sets as in Theorem 7.3. Let $n_{1}=1$. When $n_{1}, \cdots, n_{k}$ have been chosen, let $n_{k+1}$ be the least positive integer different from all of the numbers $n_{p}+n_{q}-n_{s}$, where $1 \leqq p \leqq k, 1 \leqq q \leqq k, 1 \leqq s \leqq k$. Clearly this inductive definition produces a set satisfying the condition of Theorem 7.1. In fact the number $B$ of Theorem 7.1 is 1 . It is also clear that $n_{1}<n_{2}<n_{3}<\cdots$. The set of all $n_{p}+n_{q}-n_{z}$ as above has at most $k^{3}$ members, and so $n_{k+1}$ is one of the numbers $1,2, \cdots, k^{3}+1$. Thus $n_{k+1} \leqq k^{3}+1$ and $n_{k} \leqq k^{3}(k=1,2,3, \cdots)$. Assume that $N=\mathrm{U}_{j=1}^{r} L_{j}$, where the $L_{j}$ satisfy lacunary conditions as in Theorem 7.3. At least $[k / r]$ of the numbers $n_{1}, \cdots, n_{k}$ will lie in $L_{j}$ for some $j$ depending on $k$. Writing out these $[k / r]$ numbers in numerical order, we have $1 \leqq x_{1}<x_{2}<\cdots<x_{[k / r]} \leqq n_{k} \leqq k^{3}$. Let $\lambda=\min \left(\lambda_{1}, \cdots, \lambda_{r}\right)$. Then we have $\lambda^{(k / r)-2}<\lambda^{[k / r]-1} \leqq \lambda^{[k / r]-1} x_{1} \leqq x_{[k / r]} \leqq k^{3}$, that is, $\lambda^{(k / r)-2}<k^{3}$. For any fixed $\lambda>1$ and any fixed $r$, this inequality is false for $k$ sufficiently large.

The first ten elements of $N$ are: $1,2,4,8,13,21,31,45,66,81$.

8. Extensions to arbitrary compact groups. Theorems 2.1 and 6.1 admit generalizations that are valid for an arbitrary compact (not necessarily commutative) group. Let $G$ be any such group. Let $d t, d u$, etc., denote the differential of normalized Haar measure on $G$, and let $\mathfrak{R}_{1}(G), \mathfrak{R}_{2}(G)$, and $\mathfrak{R}_{\infty}(G)$ denote the spaces of absolutely integrable, square integrable, and essentially bounded measurable functions on $G$ with respect to Haar measure, with their usual norms $\|\cdot\|_{1},\|\cdot\|_{2}$, and $\|\cdot\|_{\infty}$. Let $\mathbb{S}(G)$ denote the space of continuous 
functions on $G$ with the uniform norm, and $\mathscr{N}(G)$ the space of complex bounded Radon measures on $G$, with the variation norm.

For $x, y \in \Omega_{1}(G)$, let $x * y$ be the function defined by $x * y(t)=\int_{G} x\left(t u^{-1}\right) y(u) d u$. It is well known that $x * y \in \mathfrak{R}_{1}(G)$ and that $\mathfrak{R}_{1}(G)$ is a Banach algebra with $x * y$ as multiplication.

8.1. Let $\left\{U^{(\lambda)}\right\}_{\lambda \in \Lambda}$ be a complete set of mutually inequivalent, continuous, irreducible, unitary representations of $G$. For each $\lambda \in \Lambda$ and $t \in G$, write $U^{(\lambda)}(t)=\left\{u_{\rho \sigma}^{(\lambda)}(t)\right\}_{\rho, \sigma=1}^{s(\lambda)}$. Let $\chi^{(\lambda)}=\sum_{\rho=1}^{s(\lambda)} u_{\rho \rho}^{(\lambda)} ; \chi^{(\lambda)}$ is the character of $U^{(\lambda)}$. For $x \in \mathcal{R}_{1}(G)$ and $u_{\rho \sigma}^{(\lambda)}$ as above, let $\left(x, u_{\rho \sigma}^{(\lambda)}\right)=\int_{G} x(t) \bar{u}_{\rho \sigma}^{(\lambda)}(t) d t$. It is well known that $\left(u_{\rho \sigma}^{(\lambda)}, u_{\rho^{\prime} \sigma^{\prime}}^{\left(\lambda^{\prime}\right)}\right)=(1 / s(\lambda)) \delta_{\lambda \lambda^{\prime}} \delta_{\rho \rho^{\prime}} \delta_{\sigma \sigma^{\prime}}$, where $\delta$ is the Kronecker symbol. The PeterWeyl theorem states that for $x \in \mathbb{R}_{2}(G)$, we have

$$
\|x\|_{2}^{2}=\sum_{\lambda \in \Lambda} s(\lambda) \sum_{\rho, \sigma=1}^{s(\lambda)}\left|\left(x, u_{\rho \sigma}^{(\lambda)}\right)\right|^{2},
$$

and hence

$$
x=\sum_{\lambda \in \Lambda} s(\lambda) \sum_{\rho, \sigma=1}^{s(\lambda)}\left(x, \hat{u}_{\rho \sigma}^{(\lambda)}\right) u_{\rho \sigma}^{(\lambda)},
$$

the convergence of the series on the right being in the $\mathfrak{R}_{2}$ norm. For $x \in \mathfrak{R}_{1}(G)$ and $\alpha>0$, there are only a finite number of functions $u_{\rho \sigma}^{(\lambda)}$ such that $\left|\left(x, u_{\rho \sigma}^{(\lambda)}\right)\right|$ $\geqq \alpha$. For $x \in \mathfrak{R}_{1}(G)$, a simple calculation shows that

$$
x * u_{\rho \sigma}^{(\lambda)}=\sum_{\tau=1}^{\circ(\lambda)}\left(x, u_{\tau \rho}^{(\lambda)}\right) u_{\tau \sigma}^{(\lambda)}, \quad u_{\rho \sigma}^{(\lambda)} * x=\sum_{\tau=1}^{\rho(\lambda)}\left(x, u_{\sigma \tau}^{(\lambda)}\right) u_{\rho \tau}^{(\lambda)} .
$$

Hence

8.1 .3

$$
x * \chi^{(\lambda)}=\chi^{(\lambda)} * x=\sum_{\rho, \sigma=1}^{s(\lambda)}\left(x, u_{\rho \sigma}^{(\lambda)}\right) u_{\rho \sigma}^{(\lambda)} .
$$

8.2. It is easy to see that an element $x$ of $\mathfrak{R}_{1}(G)$ is in the center of $\mathfrak{R}_{1}(G)$ if and only if $\left(x, u_{\rho \sigma}^{(\lambda)}\right)=\delta_{\rho \sigma}\left(x, u_{11}^{(\lambda)}\right)$ for all $\lambda, \rho$, and $\sigma$. It can also be shown that for every neighborhood $W$ of the identity $e$ in $G$, there is a continuous function $h$ in the center of $\mathfrak{R}_{1}(G)$ such that $h$ is non-negative, $h$ vanishes outside of $W, \int_{G} h(t) d t=1$, and $\left(h, u_{11}^{(\lambda)}\right) \geqq 0$ for all $\lambda$. This is done by starting with an arbitrary non-negative continuous function $\varphi$ vanishing outside of a certain neighborhood $W_{1}$ of $e$, writing $\psi(t)=\int_{a \varphi}\left(u^{-1} t u\right) d u, \tilde{\psi}(t)=\psi\left(t^{-1}\right)$, and finally $h=\psi * \tilde{\psi}$. If $W_{1}$ is a small enough neighborhood of $e$ and $\varphi$ is not identically zero, then $h$ will satisfy all of the conditions above except for the trivial normalization $\int_{G} h(t) d t=1$.

For our main theorems, we require a weakened form of Fejér's summability theorem for Fourier series, as follows.

8.3. Lemma. Let $x \in \mathfrak{R}_{1}(G)$, let $\left\{\lambda_{1}, \cdots, \lambda_{n}\right\}$ be any finite subset of $\Lambda$, and let $\epsilon$ be any positive number. Then there exist a subset $\left\{\lambda_{1}, \cdots, \lambda_{m}\right\}$ of $\Lambda$ con- 
taining $\left\{\lambda_{1}, \cdots, \lambda_{n}\right\}$ and numbers $\gamma_{1}, \cdots, \gamma_{m}$ such that $1-\epsilon \leqq \gamma_{j} \leqq 1$ for $j=1,2, \cdots, n$ and for which

8.3 .1

$$
\left\|\sum_{j=1}^{m} s\left(\lambda_{j}\right) \gamma_{j} \sum_{\rho, \sigma=1}^{s\left(\lambda_{j}\right)}\left(x, u_{\rho \sigma}^{\left(\lambda_{j}\right)}\right) u_{\rho \sigma}^{\left(\lambda_{j}\right)}\right\|_{1} \leqq 2\|x\|_{1} .
$$

If $x \in \mathfrak{R}_{\infty}(G)$, then $\lambda_{1}, \cdots, \lambda_{m}$ and $\gamma_{1}, \cdots, \gamma_{m}$ as above exist such that

8.3 .2

$$
\left\|\sum_{j=1}^{m} s\left(\lambda_{j}\right) \gamma_{j} \sum_{\rho, \sigma=1}^{s\left(\lambda_{j}\right)}\left(x, u_{\rho \sigma}^{\left(\lambda_{j}\right)}\right) u_{\rho \sigma}^{\left(\lambda_{j}\right)}\right\|_{\infty} \leqq 2\|x\|_{\infty} .
$$

Proof. Choose a function $h$ as in 8.2 with the additional properties that $1-\epsilon \leqq\left(h, u_{11}^{\left(\lambda_{j}\right)}\right)$ for $1 \leqq j \leqq n$ and

$$
\|x-x * h\|_{1} \leqq \frac{1}{2}\|x\|_{1} .
$$

This can be done by choosing $W$ small enough. In view of 8.1 .2 we can find. a finite set of indices $\lambda$, say $\left\{\lambda_{1}, \cdots, \lambda_{m}\right\}$, with $m \geqq n$, for which

$$
\left.\| h-\sum_{j=1}^{m} s\left(\lambda_{j}\right) \sum_{\rho, \sigma=1}^{s\left(\lambda_{j}\right)}\left(h, u_{\rho \sigma}^{\left(\lambda_{j}\right)}\right) u_{\rho \sigma}^{\left(\lambda_{j}\right)}\right) \|_{2}<\frac{1}{2} .
$$

Write $\gamma_{j}=\left(h, u_{11}^{\left(\lambda_{j}\right)}\right)(j=1, \cdots, m)$. Then we have

$$
h_{m}=\sum_{j=1}^{m} s\left(\lambda_{j}\right) \sum_{\rho, \sigma=1}^{8\left(\lambda_{j}\right)}\left(h, u_{\rho \sigma}^{\left(\lambda_{j}\right)}\right) u_{\rho \sigma}^{\left(\lambda_{j}\right)}=\sum_{j=1}^{m} s\left(\lambda_{j}\right) \gamma_{j} \chi^{\left(\lambda_{j}\right)}
$$

and

8.3 .4

$$
\left\|h-h_{m}\right\|_{2}<\frac{1}{2}
$$

From 8.1.3, we have

$$
x * h_{m}=\sum_{j=1}^{m} s\left(\lambda_{j}\right) \gamma_{j} \sum_{\rho, \sigma=1}^{s\left(\lambda_{j}\right)}\left(x, u_{\rho \sigma}^{\left(\lambda_{j}\right)}\right) u_{\rho \sigma}^{\left(\lambda_{j}\right)} .
$$

We also have $\left\|x * h-x * h_{m}\right\|_{1} \leqq\|x\|_{1} \cdot\left\|h-h_{m}\right\|_{1} \leqq\|x\|_{1} \cdot\left\|h-h_{m}\right\|_{2}<\|x\|_{1} / 2$, and hence

$$
\left\|x * h_{m}\right\|_{1} \leqq \frac{1}{2}\|x\|_{1}+\|x * h\|_{1} .
$$

Combining 8.3.6, 8.3.5, and 8.3.3, we obtain 8.3.1.

The inequality 8.3.2 is proved in like manner. For any $h$ as in 8.2 for which $1-\epsilon \leqq\left(h, u_{11}^{\left(\lambda_{j}\right)}\right)(j=1, \cdots, n)$ and a bounded $x$, we have

$$
\|x * h\|_{\infty} \leqq\|x\|_{\infty} \text {. }
$$


We also have $\left\|x * h_{m}-x * h\right\|_{\infty} \leqq\|x\|_{\infty}\left\|h-h_{m}\right\|_{1} \leqq\|x\|_{\infty}\left\|h-h_{m}\right\|_{2}<\|x\|_{\infty} / 2$, so that

$$
\left\|x * h_{m}\right\|_{\infty} \leqq\|x * h\|_{\infty}+\frac{1}{2}\|x\|_{\infty} .
$$

Combining 8.3.7, 8.3.8, and 8.3.5, we obtain 8.3.2.

8.4. REMARK. Lemma 8.3 can obviously be extended to show that sums $\sum_{j=1}^{m} s\left(\lambda_{j}\right) \gamma_{j}(W) \sum_{\rho, \sigma=1}^{s\left(\lambda_{j}\right)}\left(x, u_{\rho \sigma}^{\left(\lambda_{j}\right)}\right) u_{\rho \sigma}^{\left(\lambda_{j}\right)}$ converge in the $\mathfrak{l}_{1}$ norm to $x$, and boundedly if $x$ is bounded: i.e., Fejér's theorem has a complete analogue for arbitrary compact groups.

We now give the generalizations of Theorems 2.1 and 6.1. To state the theorems, it is convenient to write $U$ for the set of all functions $u_{\rho \sigma}^{(\lambda)}, \lambda \in \Lambda$, $1 \leqq \rho \leqq s(\lambda), 1 \leqq \sigma \leqq s(\lambda)$, and to write elements of $U$ as $u, v, \cdots$. For $u \in U, s(u)$ denotes the number $s(\lambda)$ where $u=u_{\rho \sigma}^{(\lambda)}$. For $V \subset U$, let $c_{0}(V)$ be the Banach space of all functions $\psi$ on $V$ such that $\{v: v \in V,|\psi(v)| \geqq \alpha\}$ is finite for every $\alpha>0$, with the maximum norm. Let $b(V)$ be the Banach space of all bounded functions on $\boldsymbol{V}$, with the supremum norm. Let $l_{1}(\boldsymbol{V})$ and $l_{2}(\boldsymbol{V})$ have their usual meanings.

8.5. THEOREM. Let $\boldsymbol{V}$ be a subset of $\boldsymbol{U}$. The following properties of $\boldsymbol{V}$ are equivalent.

(i) There is a positive number $A$ such that for every finite subset $\left\{v_{1}, \cdots, v_{k}\right\}$ of $V$ and every sequence $\left\{a_{1}, \cdots, a_{k}\right\}$ of complex numbers, the inequality

$$
\sum_{j=1}^{k}\left|a_{j}\right| \leqq A \max _{t \in G}\left|\sum_{j=1}^{k} a_{j} v_{j}(t)\right|
$$

obtains.

(ii) If $x \in R_{\infty}(G)$ and $(x, u)=0$ for all $u \in U \cap V^{\prime}$, then

$$
\sum_{u \in V} s(u)|(x, u)|<\infty .
$$

(iii) For every function $\psi$ in $c_{0}(V)$, there is a function $x \in \Omega_{1}(G)$ such that $\int_{G} x(t) v(t) d t=\psi(v)$ for all $v \in V$.

(iv) For every function $f \in b(V)$, there is a measure $\mu \in \mathscr{T}(G)$ such that $\int_{G} v(t) d \mu(t)=f(v)$ for every $v \in V$.

Proof. We consider the spaces $\mathfrak{R}_{1}(G)$ and $c_{0}(V)$ and the mapping $\Phi$ of $\mathfrak{R}_{1}(G)$ into $c_{0}(V)$ such that

$$
\Phi(x)(v)=\int_{G} x(t) v(t) d t, \quad v \in V .
$$

It is clear that $\|\Phi(x)\| \leqq\|x\|_{1}$. The adjoint mapping $\Phi^{*}$ of $\Phi$ carries the adjoint space $l_{1}(V)$ into the adjoint space $\ell_{\infty}(G)$. A short calculation shows that $\Phi^{*}(\varphi)=\sum_{v \in V} \varphi(v) v$, for all $\varphi \in l_{1}(V)$. Thus $\Phi^{*}(\varphi) \in \mathbb{E}(G)$, so that $\Phi^{*}$ maps 
$h(V)$ into $\mathfrak{S}(G)$, with the uniform norm. Property (i) is obviously equivalent to the assertion that $\Phi^{*}$ has a continuous inverse.

We now prove that (i) implies (ii). Consider any $x \in \mathfrak{R}_{\infty}(G)$ such that $(x, u)=0$ for $u \in U \cap V^{\prime}$, and any finite set $\left\{u_{1}, \cdots, u_{k}\right\} \subset U$. We may suppose with no loss of generality that $\left\{u_{1}, \cdots, u_{k}\right\}$ contains all $u$ 's from a given representation $U^{(\lambda)}$ if it contains any at all: $\left\{u_{1}, \cdots, u_{k}\right\}$ $=\left\{u_{\rho, \sigma}^{\left(\lambda_{j}\right)}\right\}_{\rho, \sigma=1}^{s\left(\lambda_{j}\right)}{ }_{j=1}^{n}$. Now applying Lemma 8.3 and (i), and taking $\lambda_{1}, \cdots, \lambda_{m}$ as in Lemma 8.3, we have

$$
\begin{aligned}
& \sum_{j=1}^{n} s\left(\lambda_{j}\right) \gamma_{j} \sum_{\rho, \sigma=1}^{s\left(\lambda_{j}\right)}\left|\left(x, u_{\rho \sigma}^{\left(\lambda_{j}\right)}\right)\right| \leqq \sum_{j=1}^{m} s\left(\lambda_{j}\right) \gamma_{j} \sum_{\rho, \sigma=1}^{8\left(\lambda_{j}\right)}\left|\left(x, u_{\rho \sigma}^{\left(\lambda_{j}\right)}\right)\right| \\
& \leqq A \max _{t \in G}\left|\sum_{j=1}^{m} s\left(\lambda_{j}\right) \gamma_{j} \sum_{\rho, \sigma=1}^{8\left(\lambda_{j}\right)}\left(x, u_{\rho \sigma}^{\left(\lambda_{j}\right)}\right) u_{\rho \sigma}^{\left(\lambda_{j}\right)}(t)\right| \leqq 2 A\|x\|_{\infty} .
\end{aligned}
$$

Since $\gamma_{1}, \cdots, \gamma_{n}$ are arbitrarily close to 1 , (ii) follows.

We prove that (ii) implies (i) by showing that if (ii) holds, then $\Phi^{*}\left(l_{1}(V)\right.$ ) is a closed subspace of $\mathfrak{S}(G)$, and then repeating the argument used to show that 2.1 (ii) implies 2.1 (i).

Both (iii) and (iv) are equivalent to the assertion that $\Phi^{*-1}$ is continuous: see the proof of Theorem 2.1 .

8.6. TheOREM. Let $\boldsymbol{V}$ be a subset of $U$. The following properties of $\boldsymbol{V}$ are equivalent.

(i) There is a positive number $A$ such that for every finite subset $\left\{v_{1}, \cdots, v_{k}\right\}$ of $V$ and every sequence $\left\{a_{1}, \cdots, a_{k}\right\}$ of complex numbers, the inequality

$$
\left(\sum_{j=1}^{k}\left|a_{j}\right|^{2}\right)^{1 / 2} \leqq A \int_{G}\left|\sum_{j=1}^{k}\left(s\left(v_{j}\right)\right)^{1 / 2} a_{j} v_{j}(t)\right| d t
$$

obtains.

(ii) If $x \in \mathfrak{R}_{1}(G)$ and $(x, u)=0$ for $u \in U \cap V^{\prime}$, then

$$
\sum_{u \in V} s(u)|(x, u)|^{2}<\infty .
$$

(iii) For every $\varphi \in l_{2}(V)$, there is an $x \in \mathfrak{S}(G)$ such that

$$
(s(v))^{1 / 2} \int_{G} x(t) v(t) d t=\phi(v), \quad v \in V .
$$

(iv) For every $\varphi \in l_{2}(V)$, there is an $x \in \mathfrak{R}_{\infty}(G)$ such that

$$
(s(v))^{1 / 2} \int_{G} x(t) v(t) d t=\phi(v), \quad v \in V .
$$

Proof. We use here a mapping $\Phi$ slightly different from that used in the previous proof. For $x \in \mathbb{E}(G)$, let $\Phi(x)$ be the function on $V$ such that $\Phi(x)(v)$ 
$=(s(v))^{1 / 2} \int_{G} x(t) v(t) d t$. It is clear from 8.1.1 that $\Phi(x) \in l_{2}(V)$ and that $\|\Phi(x)\|_{2} \leqq\|x\|_{\infty}$. The mapping $\Phi^{*}$ carries $l_{2}(V)$ into $\mathfrak{M}(G)$. For $\varphi \in l_{2}(V)$, we see that the measure corresponding to $\Phi^{*}(\varphi)$ is absolutely continuous, and has differential equal to $\left(\sum_{v \in V} \varphi(v)(s(v))^{1 / 2} v(t)\right) d t$. The series $\sum_{v \in V} \varphi(v)(s(v))^{1 / 2} v$ converges in the $\mathfrak{R}_{2}$ norm, so that $\sum_{v \in V} \varphi(v)(s(v))^{1 / 2} v$ is a function in $\mathfrak{R}_{2}(G)$ and hence in $\ell_{1}(G)$. The norm in $\Phi^{*}\left(l_{2}(V)\right)$ is the $\ell_{1}$ norm, which a'grees with the norm in $\mathscr{T}(G)$. Thus $\Phi^{*}$ maps $l_{2}(V)$ into $\mathfrak{R}_{1}(G)$.

Property (i) asserts that $\Phi^{*-1}$ is continuous. Properties (iii) and (iv) are both equivalent to (i), by the arguments used in proving the corresponding assertions of Theorem 2.1.

We prove next that (i) implies (ii). Let $x$ be a function in $\Omega_{1}(G)$ such that $(x, u)=0$ for $u \in U \cap V^{\prime}$, and let $\left\{\lambda_{1}, \cdots, \lambda_{n}\right\}$ be any finite subset of $\Lambda$. Let $\left\{\lambda_{1}, \cdots, \lambda_{m}\right\}$ and $\gamma_{1}, \cdots, \gamma_{m}$ be as in Lemma 8.3 and inequality 8.3.1. Then if (i) holds, we have

$$
\begin{aligned}
\left(\sum_{j=1}^{n} s\left(\lambda_{j}\right) \gamma_{j}^{2} \sum_{\rho, \sigma=1}^{s\left(\lambda_{j}\right)} \mid(x,\right. & \left.\left.u_{\rho \sigma}^{\left(\lambda_{j}\right)}\right)\left.\right|^{2}\right)^{1 / 2} \leqq\left(\sum_{j=1}^{m} s\left(\lambda_{j}\right) \gamma_{j}^{2} \sum_{\rho, \sigma=1}^{s\left(\lambda_{j}\right)}\left|\left(x, u_{\rho \sigma}^{\left({ }^{j}\right)}\right)\right|^{2}\right)^{1 / 2} \\
& \leqq A \int_{G}\left|\sum_{j=1}^{m} s\left(\lambda_{j}\right) \gamma_{j} \sum_{\rho, \sigma=1}^{s\left(\lambda_{j}\right)}\left(x, u_{\rho \sigma}^{\left(\lambda_{j}\right)}\right) u_{\rho \sigma}^{\left(\lambda_{j}\right)}(t)\right| d t \leqq 2 A\|x\|_{1} .
\end{aligned}
$$

Property (ii) follows at once. The implication (ii) $\Rightarrow$ (i) is proved just as in the proof of Theorem 2.1.

9. Examples for Theorem 8.5. In this section, we find some sets that satisfy the conditions of Theorem 8.5. In order to obtain usable results, we restrict ourselves to subsets $V$ of $U$ on which $s(v)$ is bounded.

9.1. Lemma. Let $V$ be a subset of $U$ with the property that for every bounded function $f$ on $V$, there is a positive number $A$ such that for every finite subset $F$ of $V$, there is a measure $\nu_{F} \in \mathscr{N}(G)$ for which $\int_{G} v(t) d \nu_{F}(t)=f(v)$ for all $v \in F$ and $\left\|\nu_{F}\right\| \leqq A$. Then $V$ satisfies 8.5 (iv).

Proof. Consider $\mathfrak{T}(G)$ in its weak topology as the adjoint space of $\mathfrak{S}(G)$. Let $\odot(F)$ be the set of all $\nu \in \mathscr{T}(G)$ such that $\|\nu\| \leqq A$ and $\int_{G} v(t) d \nu(t)=f(v)$ for all $v \in F$. Plainly $\odot(F)$ is closed and bounded and is therefore compact. Also we have $\rho\left(F_{1}\right) \cap \cdots \cap \odot\left(F_{l}\right) \supset \odot\left(F_{1} \cap \cdots \cap F_{l}\right)$. The hypothesis implies that the family of all $\odot(F)$ has the finite intersection property. Therefore there is a measure $\mu \in \cap \odot(F)$, the intersection being taken over all finite subsets of $V$. This measure $\mu$ clearly satisfies 8.5 (iv), for the function $f$.

9.2. Theorem. Let $V$ be a subset of $U$ on which $s(v)$ is bounded and such that $V=U_{j=1}^{r} V_{j}$, where the $V_{j}$ are pairwise disjoint and satisfy the following condition. For a nonvoid finite subset $\left\{v_{1}, \cdots, v_{q}\right\}$ of $V_{j}$, let $\varphi=v_{1}^{\left(\delta_{1}\right)} \cdots v_{q}^{\left(\delta_{q}\right)}$, where each $v_{j}^{\left(\delta_{j}\right)}$ can be either $v_{j}$ or $\bar{v}_{j}$. Suppose that every function $\varphi$ is orthogonal to 
1 and that every function $\varphi$ is orthogonal to all $v \in V$ except in the case $q=1$, $v_{1}^{\left(\delta_{1}\right)}=v$. Then $V$ satisfies all of the conditions of Theorem 8.5.

Proof. Let $f$ be a bounded function on $V$; we may suppose with no loss of generality that $|f(v)| \leqq 1 / s(v)$ for all $v \in V$. For $F_{j}=\left\{v_{1}, \cdots, v_{q}\right\} \subset V_{j}$, let

$$
h_{F_{j}}=2 \prod_{h=1}^{q}\left(1+s\left(v_{h}\right) \Re\left(f\left(v_{h}\right) \bar{v}_{h}\right)\right) .
$$

Clearly $h_{F_{j}}$ is a non-negative function on $G$. Multiplying out, we have

$$
h_{F_{i}}=2+\sum_{h=1}^{q} s\left(v_{h}\right) f\left(v_{h}\right) \bar{v}_{h}+\sum_{\varphi} a_{\varphi} \varphi,
$$

where each $\varphi$ appearing in the second sum has the form $v_{h}(h=1, \cdots, q)$ or $v_{1}^{\left(\delta_{1}\right)} \cdots v_{k}^{\left(\delta_{k}\right)}$ with $k \geqq 2$. We obviously now have

$$
\int_{\sigma} h_{F_{j}}(t) v(t) d t=\left\{\begin{array}{cl}
f\left(v_{h}\right) & \text { if } v=v_{h} \quad(h=1,2, \cdots, q), \\
0 & \text { if } v \in V \cap V_{j}^{\prime},
\end{array}\right.
$$

and $\int_{G} h_{F_{j}}(t) d t=2$.

Let $F$ be any nonvoid finite subset of $V$, and write $F_{j}=F \cap V_{j}$. Take $h_{F_{j}}=0$ if $F_{j}$ is void, and write $h_{F}=\sum_{j=1}^{r} h_{F_{j}}$. Then we have $h_{F}$ non-negative, $\int_{G} h_{F}(t) d t \leqq 2 r$, and $\int_{G} h_{F}(t) v(t) d t=f(v)$ for all $v \in F$. Let $\nu_{F}$ be the measure on $G$ such that $d \nu_{F}(t)=h_{F}(t) d t$. Then the set of all measures $\nu_{F}$, taken over all nonvoid finite subsets $F$ of $V$, satisfies the hypotheses of Lemma 9.1 with $A=2 r$. Lemma 9.1 shows that $V$ satisfies condition 8.5 (iv) and hence all of the conditions of Theorem 8.5.

9.3. REMARK. If $G$ is commutative, then $s(u)=1$ for all $u \in U$, and the functions $u$ are simply characters of $G$. Thus Theorem 9.2 generalizes Theorem 3.2, just as Theorem 8.5 generalizes Theorem 2.1. The group $G$ in Theorem 2.1 is of course the additive group of real numbers modulo $2 \pi$.

9.4. EXAмpLE. Let $\left\{G_{\omega}\right\}_{\omega \in \Omega}$ be any nonvoid family of compact commutative groups, and let $G=P_{\omega \in \Omega} G_{\omega}$. Let $\psi_{\omega}$ be any character of $G_{\omega}$ different from 1 $(\omega \in \Omega)$, and let $\chi_{\omega}$ be the function on $G$ such that $\chi_{\omega}(t)=\psi_{\omega}\left(t_{\omega}\right)$ for each $t=\left\{t_{\omega}\right\}_{\omega \in \Omega} \in G$. Then it is clear that the set $V=\left\{\chi_{\omega}\right\}_{\omega \in \Omega}$ is a set of characters of $G$, that $V$ satisfies the independence condition of Theorem 9.2, and hence that $V$ satisfies the conditions of Theorem 8.5.

9.5. EXAmple. The Rademacher functions (see $[4$, p. 42]) have been studied as a lacunary system in one sense or another by several authors. Theorem 8.5 can be applied to these functions. Let $G$ be the group of all sequences $t=\left\{t_{1}, t_{2}, t_{3}, \cdots\right\}$, where the $t_{k}$ 's are 0 or 1 , where $t+u$ $=\left\{t_{n}+u_{n}(\bmod 2)\right\}$, and neighborhoods of 0 are sets consisting of all elements $\left\{0,0, \cdots, 0, t_{n}, t_{n+1}, \cdots\right\}$. The mapping $t \rightarrow \sum_{k=1}^{\infty} 2^{-k} t_{k}=\tau(t)$ carries $G$ onto the closed interval $[0,1]$. Let $\varphi_{n}$ be the $n$th Rademacher function, and let $D$ be the set of all $t \in G$ such that an infinite number of $t_{c}$ 's are 0 and an 
infinite number are 1. Then $\varphi_{n}(\tau(t))=(-1)^{t_{n}}$ for all $t \in D$. Haar measure on $G$ goes into Lebesgue measure on $[0,1]$ under the mapping $\tau$. The functions $(-1)^{t_{n}}$ are obviously characters of $G$; let $V$ be the set of all these characters.

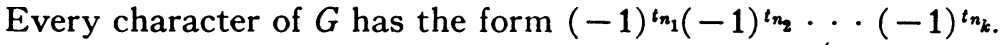

It can be shown simply that for every sequence $\left\{a_{1}, \cdots, a_{k}\right\}$ of complex numbers, the inequality

9.5 .1

$$
\sum_{j=1}^{k}\left|a_{j}\right| \leqq 2 \max _{t \in G}\left|\sum_{j=1}^{k} a_{j}(-1)^{t_{j}}\right|
$$

obtains; also

9.5 .2

$$
\sum_{j=1}^{k}\left|a_{j}\right| \leqq 2 \max _{0 \leqq \nu \leqq 1}\left|\sum_{j=1}^{k} a_{,} \phi_{j}(y)\right| .
$$

Inequality 9.5 .1 is simply condition 8.5 (i) for the group $G$ and the set of characters $V$. Hence conditions 8.5 (ii)-8.5 (iv) hold for the group $G$ and the set of characters $V$. (Condition 8.5 (iv) can also be obtained easily from 9.4, since $G$ is the product of two-element groups.) Conditions 8.5 (ii) and 8.5 (iii) for $G$ and $V$ can be translated into statements about the Rademacher functions, if we use the mapping $\tau$. These are well known: (see $[4, \mathrm{pp} .254-$ 256]).

Condition 8.5 (iv) cannot be immediately translated into a statement about the Rademacher functions, because the mapping $\tau$ of $G$ onto $[0,1]$ is not one-to-one, so that a general measure $\mu$ on $G$ has no unique "image" on $[0,1]$. Furthermore, for a general measure $\lambda$ on $[0,1]$, the integral $\int_{[0,1]} \varphi_{n}(y) d \lambda(y)$ is affected by the values of $\varphi_{n}$ at dyadic rational numbers (as does not happen if $\lambda$ is Lebesgue measure); for this reason, we must change the standard definition of $\varphi_{n}$ at some dyadic rationals. For a bounded sequence $f(n)$, condition 8.5 (iv) shows that there is a measure $\mu$ on $G$ (even a positive measure) such that $f(n)=\int_{G}(-1)^{t_{n}} d \mu(t)(n=1,2,3, \cdots)$. Let $E$ be the set of all $t \in G$ such that $t_{n}$ is ultimately 0 , and write $F=D^{\prime} \cap E^{\prime}$. For $t \in E$, we write $t^{\prime}=t+\{1,1,1, \cdots\}$, obtaining a one-to-one mapping of $E$ onto $F$. Let $\nu$ be the Borel measure on $G$ such that $\nu(A)=\mu(A)$ for $A \subset D$, $\nu(t)=\mu(t)-\mu\left(t^{\prime}\right)$ for $t \in E$, and $\nu(t)=0$ for $t \in F$. Since $(-1)^{t_{n}^{\prime}}=-(-1)^{t_{n}}$, we have

$$
\begin{aligned}
f(n)=\int_{G}(-1)^{t_{n}} d \mu(t) & =\int_{D}(-1)^{t_{n}} d \nu(t)+\sum_{t \in E}(-1)^{t_{n}} \mu(t)-\sum_{t^{\prime} \in F}(-1)^{t_{n}} \mu\left(t^{\prime}\right) \\
& =\int_{D}(-1)^{t_{n}} d \nu(t)+\sum_{t \in E}(-1)^{t_{n}} \nu(t)=\int_{D \cup E}(-1)^{t_{n}} d \nu(t) .
\end{aligned}
$$

Let $\lambda$ be the measure on $[0,1]$ such that $\lambda(A)=\nu\left(\tau^{-1}(A)\right)$. Since $\tau$ is one-toone on $D \cup E$ and $\nu$ is 0 on $F$, we have 


$$
f(n)=\int_{[0,1[}(-1)^{y_{n}} d \lambda(y),
$$

where $y_{n}$ is the $n$th entry in the dyadic expansion for $y$ that contains infinitely many zeros. Let $\tilde{\varphi}_{n}$ be the function such that $\tilde{\varphi}_{n}(y)=\lim _{z \rightarrow y+} \varphi_{n}(z)$. Then we have $\tilde{\varphi}_{n}(y)=(-1)^{y_{n}}$ for all $y \in[0,1[$, and hence

$$
f(n)=\int_{[0,1]} \tilde{\varphi}_{n}(y) d \lambda(y) \quad(n=1,2,3, \cdots) .
$$

We note finally that there is an obvious analogue of Theorem 7.1 valid for all compact commutative groups (see also the extension to noncommutative compact groups in [3]).

Added in proof. Professor Walter Rudin has pointed out that the set constructed in 7.4 has been discussed earlier. See A. Stöhr, Gelöste und ungelöste Fragen über Basen der natürlichen Zahlenreihe, I, II. J. Reine Angew. Math. vol. 194 (1955) pp. 40-65, 111-143, reference on p. 134. In connection with $\$ 3$, see also S. B. Stečkin, $O b$ absolyutnot shodimosti ryadov Fur'e (tret'e soobక̌̌enie), Izv. Akad. Nauk SSSR, Ser. Mat. vol. 20 (1956) pp. 385-412.

\section{REFERENCES}

1. Stefan Banach, Théorie des opérations linéaires, Monografje Matematyczne, vol. I, Warszawa, 1932.

2. - Über einige Eigenschaften der lakünaren trigonometrischen Reihen, Studia Math. vol. 2 (1930) pp. 207-220.

3. Sigurdur Helgason, Duality and Fourier analysis on compact groups, to appear.

4. Stefan Kaczmarz and Hugo Steinhaus, Theorie der Orthogonalreihen, Monografje Matematyczne, vol. VI, Warszawa-Lw6w, 1935.

5. S. Sidon, Über orthogonale Entwicklungen, Acta Univ. Szeged., vol. 10 (1941-1943) pp. 206-253.

6. - Verallgemeinerung der in meiner Arbeit "Ein Satz über trigonometrische Polynome mit Lücken und seine Anwendung in der Theorie der Fourier-Reihen", J. Reine Angew. Math. vol. 163, bewiesene Sätze, vol. 166 (1931) pp. 62-63.

7. Antoni Zygmund, Trigonometrical series, Monografje Matematyczne, vol. V, WarszawaLwów, 1935.

The University of Washington, Seattle, Washington 